\title{
FUSE and the Quest for High-Resolution Spectroscopy in the Far Ultraviolet
}

\author{
H. Warren Moos \\ Department of Physics and Astronomy, The Johns Hopkins University, \\ Baltimore, MD 21218 USA
}

\begin{abstract}
The Far Ultraviolet Spectroscopic Explorer (FUSE) instrument covers the spectral range $912-1187 \AA$ with a resolving power of 15,000 to 20,000 . This spectral region provides unique access for the study of many atomic and ionic species found in the interstellar medium, intergalactic medium, stars, and extragalactic objects. This paper summarizes the status of the mission and then discusses the need for higher resolution spectroscopy. Although the FUSE instrumental resolution is sufficient to separate most species, it usually it is not adequate for analyzing the gas velocity structure in detail. Implications for future missions are discussed.
\end{abstract}

\section{The FUSE Mission}

The Far Ultraviolet Spectroscopic Explorer (FUSE) Mission was developed and is operated by the Johns Hopkins University for NASA with participation by the Canadian Space Agency and the Centre National d'Etudes Spatiales in France. Covering the far ultraviolet from 905 to $1187 \AA$ with a resolution $\sim 20,000$ (15 to $20 \mathrm{~km} \mathrm{~s}^{-1}$ ), it complements the intermediate resolution capabilites of the Space Telescope Imaging Spectrograph. FUSE was launched 1999 June 24 into a $765 \mathrm{~km}$ circular orbit with an instrument package consisting of four coaligned prime focus telescopes, each imaged on the entrance aperture of a Rowland mount spectrograph. For a more detailed discussion of the FUSE mission see Moos et al. (2000) and Sahnow et al. (2000). The Prime Science Mission began 1999 December 1 and ended 2003 March 31. The Mission is now in the Extended Mission Phase.

The Mission has been quite successful. As of 2002 August, $\sim 30$ million seconds of data have been obtained for more than 1700 different objects. A broad range of science has been studied ranging from solar-system planets, e.g., Krasnopolsky \& Feldman (2001) to the intergalactic medium, e.g., Shull, Tumlinson, \& Giroux (2003). We will not attempt to survey the rich and growing literature reporting results from this mission. Rather, we will examine the need for a mission with increased spectral resolution and the technical challenges associated with such a mission.

\section{High-Resolution Spectroscopy in the Far Ultraviolet}

A major limitation in the extension of far ultraviolet studies to more complex sightlines is spectral resolution. Spectroscopic studies in the far ultraviolet are 


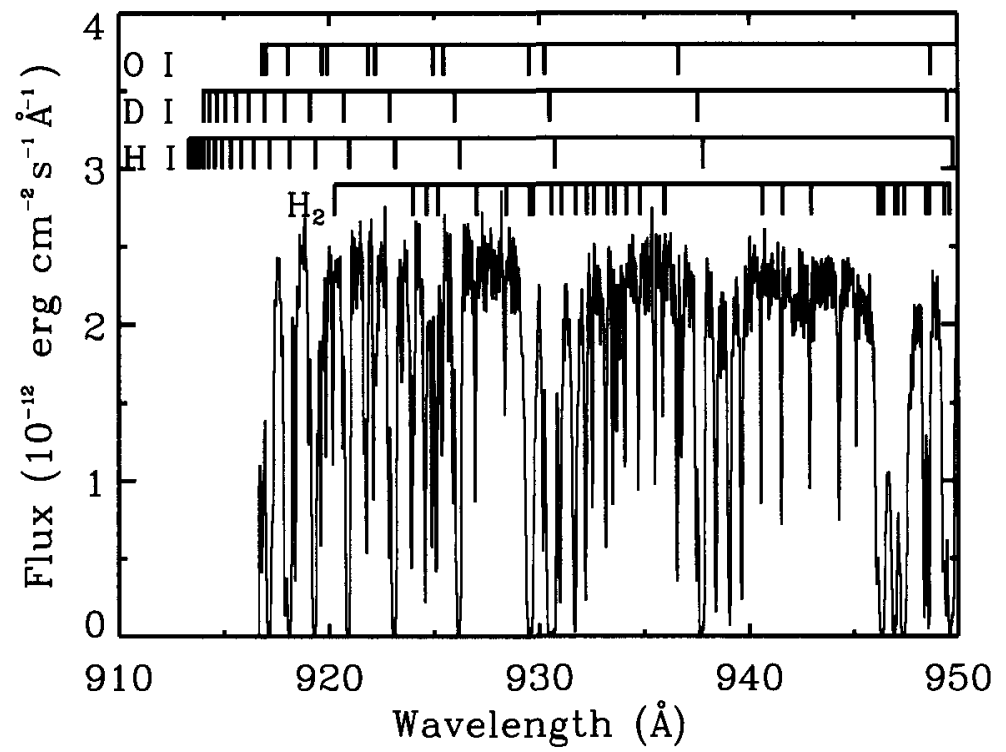

Figure 1. A portion of the FUSE spectrum of PG0038 showing numerous transitions of $\mathrm{HI}, \mathrm{D}$ I, O I and $\mathrm{H}_{2}$. At the FUSE resolution, $\mathrm{H}_{2}$ is often blended with other species. Courtesy of C. Oliveira.

powerful because the region provides access, often unique, to diagnostic transitions in numerous atoms, ions and molecules in the interstellar medium, intergalactic medium, stars, and extragalactic objects. This spectral region is rich, containing strong transitions for Hi, Di, O I, O IV, Ni, NiI, NiII, C III, Ar I, Fe II, $\mathrm{H}_{2}$, and many other species. However, the presence of so many transitions in this region can lead to blends. The FUSE resolution of $\sim 15 \mathrm{~km} \mathrm{~s}^{-1}$ is sufficient to remove most blends between transitions, with the notable exception of the numerous transitions of $\mathrm{H}_{2}$, which are both a blessing and a curse. See Figure 1. However, the FUSE resolution is inadequate for understanding the velocity structure of the gas along a sight line in interstellar medium studies. Generally, moderate resolution will make it difficult to determine whether a line is saturated. In addition, for finite signal to noise, unsaturated weak lines with low $\mathrm{b}$ values will be hard to detect. As a result, there is increased uncertainty and less knowledge about systematic errors for complex sightlines.

Is it possible to construct a general purpose FUV observatory with spectral resolution of 1 to $3 \mathrm{~km} \mathrm{~s}^{-1}$ ? The answer is yes, but there are significant technical challenges, both in terms of the telescope quality and the transmission of the optical system. To provide enough photons for sensitive high-resolution measurements, the size of the telescope mirror must be much larger than the $0.4 \mathrm{~m}$ FUSE telescopes. This leads to demands on both the optical quality and overall length of the instrument package.

First, note that for a high-resolution spectrum, the geometric area of the detector pixels must be as small as possible or the background signal from cosmic rays and scattered light will be too high for sensitive measurements. The smallest possible size of the pixel is set by the entrance aperture to the spectrograph, 
which in turn is set by the point spread function of the optics and the jitter in the spacecraft pointing. Thus, for a modest $1 \mathrm{~m}$ telescope with a focal length of $15 \mathrm{~m}, 50 \mu \mathrm{m}$ in the focal plane (equivalent to 2 pixels) corresponds to $0.7^{\prime \prime}$. The point spread function and spacecraft pointing jitter need to be much less than this, a few times the telescope optical diffraction limit at $5000 \AA\left(0.12^{\prime \prime}\right)$. This requirement is important, but does not appear to be a showstopper.

Second, note that the relatively simple FUSE design (a prime focus telescope and a Rowland circle spectrograph) with only two reflecting surfaces probably will not be adequate for reasonably high sensitivity. An instrument with a modest one meter telescope would be $\sim 20 \mathrm{~m}$ in length, too long to fit inside most launch vehicles. In addition, the FUSE instrument design carries unremovable residual abberrations that could limit the resolution and increase the background signal. In order to overcome the double disadvantages of long length and residual aberrations, a second reflection in both the the telescope and instrument are necessary.

This second reflection is perfectly acceptable if the system operates above $1000 \AA$, where high reflectivity coatings exist. Copernicus, for example, used a Cassegrain telescope (Rogerson et al. 1973). However, at shorter wavelengths down to the $\mathrm{H}$ I photoionization cut off at $912 \AA$, reflectivies are $\sim 0.3$ and about one photon in a thousand falling on the telescope will be detected.

The obvious solution is to restrict instruments to wavelengths above $1000 \AA$, but at the cost of losing many interesting transitions including the most useful ones for D I. Alternatively, we can seek new coatings and other techniques that will permit us to work at these wavelengths. In any case, the limitations set by reflectivity must be taken into account in planning high spectral resolution missions for the far ultraviolet.

Acknowledgments. This work is based in part on data obtained for the Guaranteed Time Team by the NASA-CNES-CSA FUSE mission operated by the Johns Hopkins University. Financial support has been provided by NASA contract NAS5-32985.

\section{References}

Krasnopolsky, V. A. \& Feldman, P. D. 2001, Science, 294, 1914

Moos, H. W. et al. 2000, ApJ, 538, L1

Rogerson, J. B., et al. 1973, ApJ, 181, L97

Sahnow, D. J. et al. 2000, ApJ, 538, L7

Shull, J. M., Tumlinson, J., \& Giroux, M. L. 2003, ApJ, 594, L107 